\title{
Weakly dispersive modal pulse propagation in the North Pacific Ocean
}

\author{
Ilya A. Udovydchenkov ${ }^{a)}$ \\ Applied Ocean Physics and Engineering Department, Woods Hole Oceanographic Institution, Woods Hole, \\ Massachusetts 02543 \\ Michael G. Brown \\ Rosenstiel School of Marine and Atmospheric Science, University of Miami, Miami, Florida 33149 \\ Timothy F. Duda \\ Applied Ocean Physics and Engineering Department, Woods Hole Oceanographic Institution, Woods Hole, \\ Massachusetts 02543 \\ Peter F. Worcester and Matthew A. Dzieciuch \\ Scripps Institution of Oceanography, University of California at San Diego, La Jolla, California 92093 \\ James A. Mercer and Rex K. Andrew \\ Applied Physics Laboratory, University of Washington, Seattle, Washington 98105 \\ Bruce M. Howe \\ Department of Ocean and Resources Engineering, University of Hawai' i at Manoa, Honolulu, Hawaii 96822 \\ John A. Colosi \\ Department of Oceanography, Naval Postgraduate School, Monterey, California 93943
}

(Received 28 March 2013; revised 9 August 2013; accepted 26 August 2013)

\begin{abstract}
The propagation of weakly dispersive modal pulses is investigated using data collected during the 2004 long-range ocean acoustic propagation experiment (LOAPEX). Weakly dispersive modal pulses are characterized by weak dispersion- and scattering-induced pulse broadening; such modal pulses experience minimal propagation-induced distortion and are thus well suited to communications applications. In the LOAPEX environment modes 1,2 , and 3 are approximately weakly dispersive. Using LOAPEX observations it is shown that, by extracting the energy carried by a weakly dispersive modal pulse, a transmitted communications signal can be recovered without performing channel equalization at ranges as long as $500 \mathrm{~km}$; at that range a majority of mode 1 receptions have bit error rates (BERs) less than $10 \%$, and $6.5 \%$ of mode 1 receptions have no errors. BERs are estimated for low order modes and compared with measurements of signal-to-noise ratio (SNR) and modal pulse spread. Generally, it is observed that larger modal pulse spread and lower SNR result in larger BERs. (C) 2013 Acoustical Society of America. [http://dx.doi.org/10.1121/1.4820882]
\end{abstract}

PACS number(s): 43.30.Bp, 43.60.Ac, 43.60.Dh, 43.60.Ek [AMT] Pages: 3386-3394

\section{INTRODUCTION}

Certain modal pulses (broadband distributions of energy with fixed mode number) exhibit little dispersionand scattering- induced pulse broadening (Brown and Udovydchenkov, 2013; Udovydchenkov and Brown, 2008). Such modal pulses are referred to here as weakly dispersive modal pulses. Using communications terminology, a signal consisting of a sequence of symbols (sometimes referred to as bits or digits) that is carried by a weakly dispersive modal pulse exhibits little inter-symbol interference (ISI); weakly dispersive modal pulses are thus potentially useful in communications applications. The utility of weakly dispersive modal pulses in communications applications is investigated experimentally in this paper. Specifically, data collected during the long-range ocean acoustic propagation experiment

\footnotetext{
a) Author to whom correspondence should be addressed. Electronic mail: ilya@whoi.edu
}

(LOAPEX) is analyzed here to investigate whether a transmitted communications signal can be recovered at ranges of 50,250 , and $500 \mathrm{~km}$ without performing channel equalization by extracting the signal carried by a weakly dispersive modal pulse.

Details of the LOAPEX experiment, which was conducted in the eastern North Pacific Ocean in 2004, are described in Mercer et al. (2005, 2009). The data analyzed here consists of wave fields excited by broadband transmissions in the 50 to $100 \mathrm{~Hz}$ band from a ship-suspended compact source, and which were recorded on a shallow vertical line array (SVLA) of hydrophones. The array, which was centered near the sound channel axis, had 40 receiving elements with $35 \mathrm{~m}$ spacing, covering depths between approximately 350 and $1750 \mathrm{~m}$. The transmitted signals were phase-coded $m$-sequences, 1023 digits long with $75 \mathrm{~Hz}$ carrier frequency, and two cycles of the carrier frequency per digit. The receptions were sampled at $1200 \mathrm{~Hz}$. In most applications involving $m$-sequences, match-filtering (pulse 
compression) of the recorded signals is performed to obtain the impulse response while simultaneously improving the signal-to-noise ratio (SNR). In contrast, in this paper no pulse compression is performed. Instead, the $m$-sequence transmissions are treated as a binary communication sequence; we investigate whether this binary information can be recovered at the receiving array without relying on knowledge of the transmitted signal. In this paper only transmissions from stations T50, T250, and T500 (approximately $44.7,244.7$, and $484.7 \mathrm{~km}$ away from the SVLA, respectively) are considered. The design of the SVLA allows resolution of the lowest ten or so normal modes at $75 \mathrm{~Hz}$. Here we consider only near-axial transmissions (source at approximately $800 \mathrm{~m}$ depth) because those transmissions strongly excite the lowest order modes, which are the focus of this paper.

Channel equalization is not considered in this paper, primarily because a discussion of that topic would detract from our focus on some interesting propagation physics. In other words, this paper should be thought of as a paper about propagation physics that happens to be motivated by communications applications. From a communications perspective the question investigated here-What is the maximum range at which a communications signal can be recovered without performing channel equalization?-is both interesting and important. We note also in this regard that the LOAPEX experiment was not designed as a communications experiment. It is of course possible to use one or more $m$-sequence receptions, together with knowledge of the transmitted $m$-sequence, to train an equalizer, and then apply that equalizer to subsequent $m$-sequence receptions in an attempt to recover the $m$-sequence. That procedure will not be further discussed.

Prior studies of underwater communication at long range in the deep ocean include those of Freitag and Stojanovic (2001), Song et al. (2009), and Song and Dzieciuch (2009). Unlike the present study, those efforts focused on some form of adaptive equalization. We note also that Morozov et al. (2008) have previously argued that it may be advantageous to perform mode-processing prior to performing equalization. That suggestion is supported by the results presented here, with the caveat that we recommend focusing on the special class of modal pulses that qualify, approximately at least, as weakly dispersive.

The remainder of the paper is organized as follows. In Sec. II weakly dispersive modal pulses are briefly described and the advantages of using these pulses in communication applications are noted. Section III outlines the processing algorithm used in the data analysis, which allows estimation of bit error rates (BERs). Section IV presents the results of this study and summarizes the observed BER trends as a function of transmission range and mode number. In Sec. V the effects of SNR and modal pulse spread on BER are considered. Conclusions are given in Sec. VI.

\section{WEAKLY DISPERSIVE MODAL PULSES}

The properties of weakly dispersive modal pulses and their relevance to underwater communication applications are described by Brown and Udovydchenkov (2013). A brief summary is given here. Modal pulses are broadband contributions to a wave field corresponding to a fixed mode number. The term weakly dispersive modal pulse is used here to describe a modal pulse which exhibits only small dispersion- and scattering-induced distortion. Dispersion- and scattering-induced contributions to modal pulse time spreads (Udovydchenkov and Brown, 2008) are $2 \pi\left(\Delta f / f_{0}\right)(|\beta(I)| I /$ $R(I)) r$ and $4 \pi^{3 / 2}(B / 3)^{1 / 2}(|\beta(I)| / R(I)) r^{3 / 2}$, respectively. Here $f_{0}$ is the center frequency, $\Delta f$ is the bandwidth, $r$ is the range, $\beta(I)$ is the waveguide invariant, $R(I)$ is the ray or mode double-loop distance, $I$ is the action variable, and $B$ is the diffusivity of energy in action (primarily due to internalwave-induced sound speed fluctuations). The action, frequency, and mode number $m$ satisfy an asymptotic quantization condition; in typical deep ocean environments $2 \pi I=m-1 / 2, m=1,2, \ldots$. Weakly dispersive modal pulses can be of two types. The first type consists of the lowest order modes. Low-order modes have small $I$ and hence small dispersive time spreads. In deep ocean environments low order modes have small scattering-induced time spreads because near-axial internal-wave-induced sound speed fluctuations (and hence also typical values of $B$ ) are small; see Virovlyansky et al. (2007), Makarov et al. (2010), and Udovydchenkov et al. (2012) for a more detailed discussion of near-axial sound scattering. The second type of weakly dispersive modes consists of those modes for which the waveguide invariant parameter $\beta$ is close to zero (Brown and Udovydchenkov, 2013). The corresponding modal pulses qualify as weakly dispersive because both the dispersiveand scattering-induced contributions to time spreads are proportional to $\beta$.

In the mid-latitude eastern North Pacific Ocean environment modes with $\beta=0$ are typically those with mode numbers between 20 and 30 at frequencies near $75 \mathrm{~Hz}$ (Udovydchenkov et al., 2012). Unfortunately, those modes are not resolved by the SVLA. Thus, it is not possible to test their utility for communication applications using LOAPEX measurements. For that reason, only the first type of weakly dispersive modal pulses is considered in this paper.

As we have noted, dispersive- and scattering-induced contributions to modal pulse time spreads grow with transmission range approximately like $r$ and $r^{3 / 2}$, respectively. Also, dispersive spreads are proportional to $m-1 / 2$. These dependencies on $r$ and $m$ reveal that weakly dispersive conditions are better satisfied at short $r$ and small $m$. At short ranges, the first few (corresponding to the lowest $m$-values) modal pulses are expected to behave like weakly dispersive modal pulses, while at larger ranges, only the $m=1$ modal pulse is expected to behave like a weakly dispersive modal pulse. Distortions of modal pulses with $m>1$ due to dispersion and scattering are expected to be stronger at long ranges, than the distortions of the $m=1$ modal pulse. These expectations will be tested using LOAPEX observations.

Figure 1 illustrates, using LOAPEX observations, why low order modal pulses are useful for communication at long ranges. In this figure the upper row shows absolute wave field intensities at three fixed ranges as a function of depth and time. The transient source used to produce that figure, 
$\mathrm{T} 50$
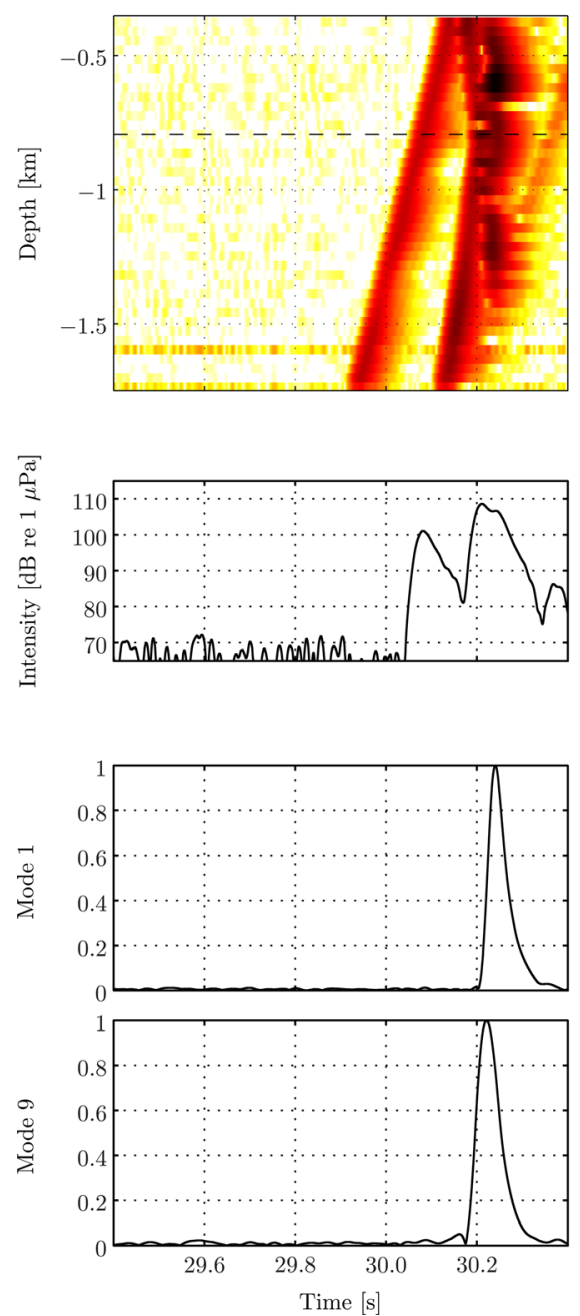

$\mathrm{T} 250$

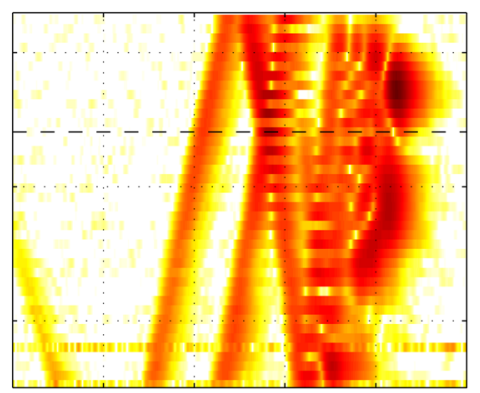

Wave field intensities

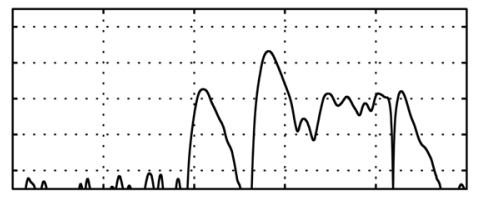

Relative mode amplitudes
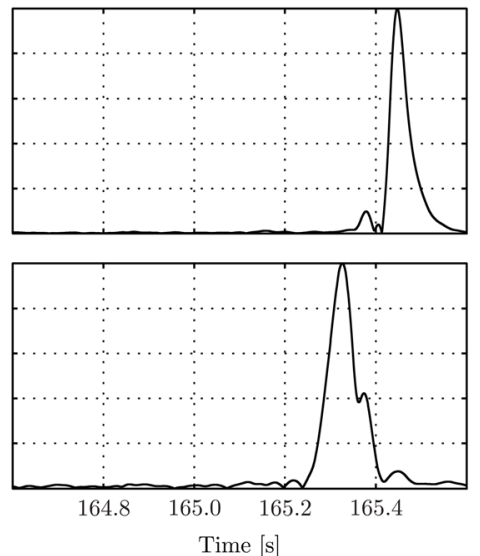

$\mathrm{T} 500$
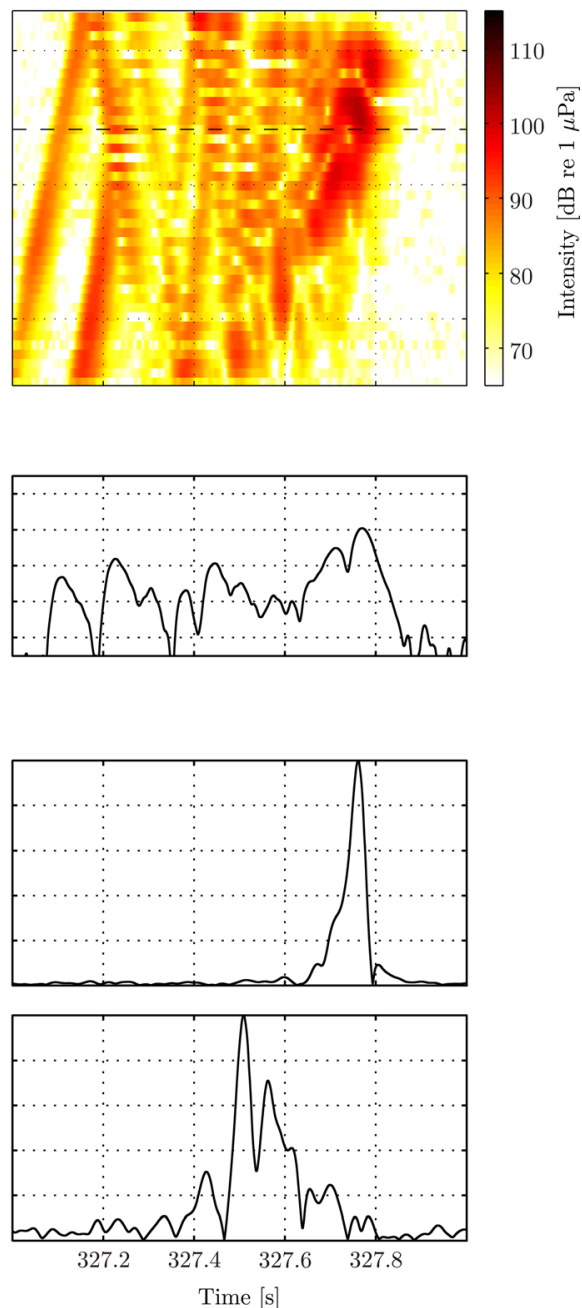

FIG. 1. (Color online) (Top row) LOAPEX wave field intensities after pulse compression as a function of time and depth for T50, T250, and T500 transmissions. (Second row) Wave field intensities as a function of time recorded at approximately $800 \mathrm{~m}$ depth corresponding to the receptions shown in the upper row. (Third row) Range evolution of an $m=1$ modal pulse. (Bottom row) Range evolution of an $m=9$ modal pulse. Normalized modal amplitudes are shown for both modal pulses.

consisting of two cycles of a $75 \mathrm{~Hz}$ carrier, may be thought of as a single digit of a communications signal. The second row shows wave field intensities measured on a hydrophone at $800 \mathrm{~m}$ depth, which is near the sound channel axis. It is clear that at this or any other depth the arrival structure is complicated by the presence of many multipath arrivals, associated pulse broadening due to scattering, and interference of energy propagating along different paths. From a communications standpoint, these effects will result in strong ISI, making interpretation of a received communications signal difficult. From a modal perspective, most modal pulses are subject to strong dispersion- and scatteringinduced pulse broadening, which also leads to strong ISI. But weakly dispersive modal pulses are special in this regard. This is illustrated in the two bottom rows of Fig. 1, which show the range evolution of modal pulses corresponding to the $m=1$ mode (third row) and the $m=9$ mode (bottom row). The $m=1$ modal pulse exhibits little temporal spreading (at least within $20 \mathrm{~dB}$ of the peak) as range increases, because that is a weakly dispersive modal pulse. The propagation-induced modal pulse broadening $\Delta t$ for mode $m=1$ is approximately $0.03 \mathrm{~s}$ at T50, $0.03 \mathrm{~s}$ at T250, and approximately $0.04 \mathrm{~s}$ at T500 [see Sec. V for the definition of propagation-induced modal pulse broadening and also see Sec. IV (B) in Udovydchenkov et al. (2012) for details on estimation of modal group time spreads]. In contrast, the temporal spread of the $m=9$ modal pulse increases significantly with increasing range. The estimated propagation-induced modal pulse broadenings for mode $m=9$ are $0.04 \mathrm{~s}$ at $\mathrm{T} 50,0.07 \mathrm{~s}$ at $\mathrm{T} 250$, and $0.23 \mathrm{~s}$ at $\mathrm{T} 500-$ a factor of 6 increase at T500. From a communications perspective, a consequence is that the $m=9$ modal pulse will exhibit much more ISI than the $m=1$ modal pulse.

\section{DATA PROCESSING}

We turn our attention now to testing, using LOAPEX observations, the expectation that weakly dispersive modal pulses are capable of carrying information to long range without significant ISI. The algorithm used to estimate BERs is now described. It is emphasized that our objective is to test 
the just-stated expectation, rather than to develop an optimal processing algorithm for communications applications.

After all timing and motion corrections were applied, mode filtering of acoustic wave fields was performed. Details are as described in Udovydchenkov et al. (2012) except that the match-filtering step was omitted here because the transmitted $m$-sequence is treated as a communication sequence. Using this process, carrier-modulated amplitudes $a_{m}(t)$ of the first ten modal pulses were constructed. Higherorder modes were not considered because they are not resolved by the SVLA. The challenge is to recover the transmitted binary sequence from a modal pulse $a_{m}(t)$. To extract the binary sequence from $a_{m}(t)$ the following procedure was used. First, the signal was bandpass filtered between 50 and $100 \mathrm{~Hz}$ and then complex demodulated to baseband. Instantaneous phase $Y_{m}(t)$ and envelope signal $A_{m}(t)$ time series were computed for each transmission using a zero-phase forward-and-reverse fifth order low pass Butterworth filter (Parks and Burrus, 1987; Oppenheim et al., 1999). Discrete samples of the phase $Y_{m}(t)$ (sampled at $1200 \mathrm{~Hz}$ ) were grouped into bins containing 32 samples (one digit is two cycles of the carrier; in the signal sampled at 16 times the carrier one digit contains 32 samples), and values within each bin were averaged. Because the transmitted sequence was a binary sequence, only the sign was retained after averaging. (For convenience we shall refer to the bits as + and bits, corresponding to the sign of the phase modulation angle of the transmitted binary sequence.) Binary sequences derived from each transmission for each $m$-value were compared with the transmitted sequence bit-by-bit. The BER is the fraction (often expressed as a percentage) of the 1023 transmitted bits that are incorrectly identified. The zerocrossings of $Y_{m}(t)$ identify the times at which the phase polarity of successive incoming digits is reversed. The number of samples between any two zero-crossings should be a multiple of 32 .

To implement this algorithm one needs to synchronize the incoming signal with the binary sequence. In other words, it is necessary to find the reference point in time at which a digit begins. Two considerations need to be taken into account. First, it is necessary to know how to group samples into bins of 32, i.e., to identify which of the 32 samples is the closest to the beginning of the digit. This can be accomplished by circularly shifting the received signal by $k$ samples, where $k$ is an integer between 0 and 31 . In practice one also needs to make sure that the synchronization time is not off by more than one digit, so in the actual implementation we varied $k$ between -32 and 64 . The second problem is to synchronize the initial phase, because the beginning of a digit, in general, does not coincide with a sampling point. This can be accomplished by shifting the phase of the signal by $\varphi_{0}$, which can vary between $-\pi$ and $\pi$. We did not attempt to find an efficient method to estimate $k$ and $\varphi_{0}$ (which likely can be done from an analysis of incoming receptions). Instead, a brute force search that minimizes BERs was implemented to determine $k$ and $\varphi_{0}$ for each $a_{m}(t)$.

The main steps involved in the implementation of the demodulation algorithm are illustrated in Fig. 2. This figure shows three examples of mode 1 modal pulses at ranges of approximately $50 \mathrm{~km}$ (top panel), $250 \mathrm{~km}$ (middle panel), and $500 \mathrm{~km}$ (bottom panel). The modal pulse $a_{1}(t)$ is shown with a thin solid line, and the phase function $Y_{1}(t)$ is shown with a thick solid line. The idealized transmitted square wave bit stream is shown with a thick gray line, and digits recovered from data are shown with dots. In the examples shown in Fig. 2, at all three ranges, the first 75 digits of the transmitted $m$-sequence were recovered with no errors. In fact, in the three examples shown all 1023 digits were recovered with no errors.

\section{RESULTS}

The results of processing all $75 \mathrm{~Hz}$ T50, T250, and T500 LOAPEX transmissions are summarized in Table I. That table shows BERs for all transmissions for the first ten modes. Three sets of BERs are listed, corresponding to processing each transmission separately, coherent averaging over $5 \mathrm{~min}$ (over 11 transmissions), and coherent averaging over $15 \mathrm{~min}$ (over 33 transmissions). Five minute averaging was chosen as a conservative estimate of the coherence time for these signals, and was also based on convenience (each file contains 5 min of data). Fifteen minute averaging was chosen as the longest interval that could reasonably be processed coherently; because of the recording schedule, averaging over longer times would often require averaging transmissions more than $1 \mathrm{~h}$ apart. For each transmission scenario four sets of results are shown. Those are the number of transmissions with BERs: Equal to zero; between $0 \%$ and 1\%; between $1 \%$ and $10 \%$; and higher than $10 \%$. Table I shows, for example, that among 330 individual T50 receptions of mode 1 , all receptions have BERs less than $10 \%$, among which 291 have zero BER, 32 have BERs between $0 \%$ and $1 \%$, and 7 transmissions have BERs between $1 \%$ and $10 \%$. Table I shows that low BERs are achievable at ranges as long as $500 \mathrm{~km}$. In fact, for mode 1 about $6.5 \%$ of transmissions have zero BERs and around $72 \%$ of transmissions have BERs less than $10 \%$.

Two important trends can be seen in Table I. First, BERs increase with increasing range. And second, BERs generally increase as mode number increases. At $50 \mathrm{~km}$, BERs are small for $m \leq 3$, substantially larger for modes 4 and 5 , and very large for $m \geq 6$. At $250 \mathrm{~km}$, BERs are clearly lowest for $m=1$, and significantly larger for modes 4 and 5 than at T50. At T500 most error-free transmissions occur for $m=1$, but many transmissions for all $m \leq 3$ have BERs less than $10 \%$. In contrast, at T500 BERs are seen to be very large for all $m \geq 4$. All these observations are consistent with the expectations stated earlier, based on theoretical arguments. These results are also consistent with prior data analysis [see Fig. 3(c) in Udovydchenkov et al. (2012)], which suggests that three lowest number modes are expected to be weakly dispersive: Mode 1 due to a small value of the action $I$, and modes 2 and 3 due to a small absolute value of the waveguide invariant $\beta$. Note also that short time coherent averaging generally helps (presumably by increasing SNR), but the trends just noted hold for both individual receptions and coherently averaged receptions. 


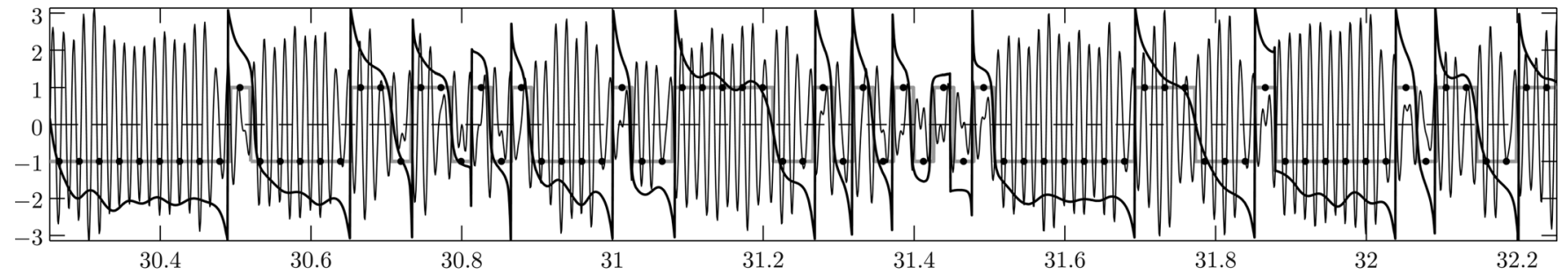

$\mathrm{T} 250$

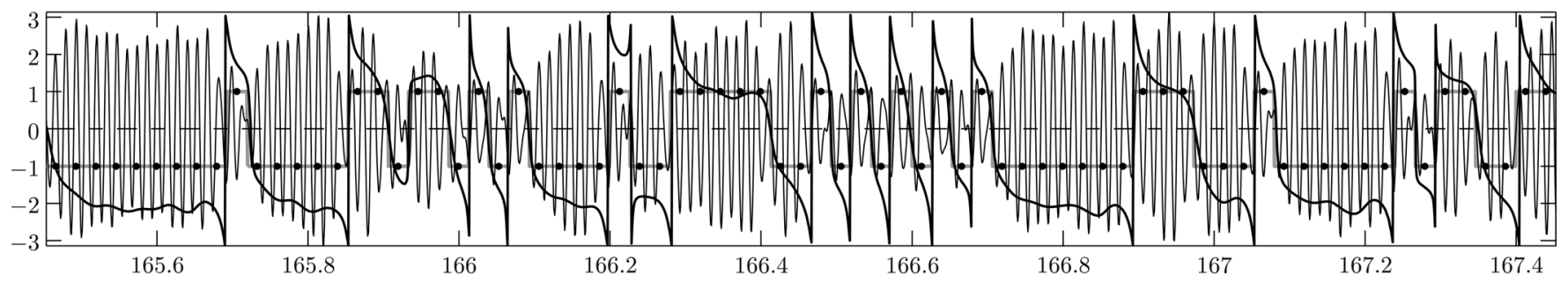

$\mathrm{T} 500$

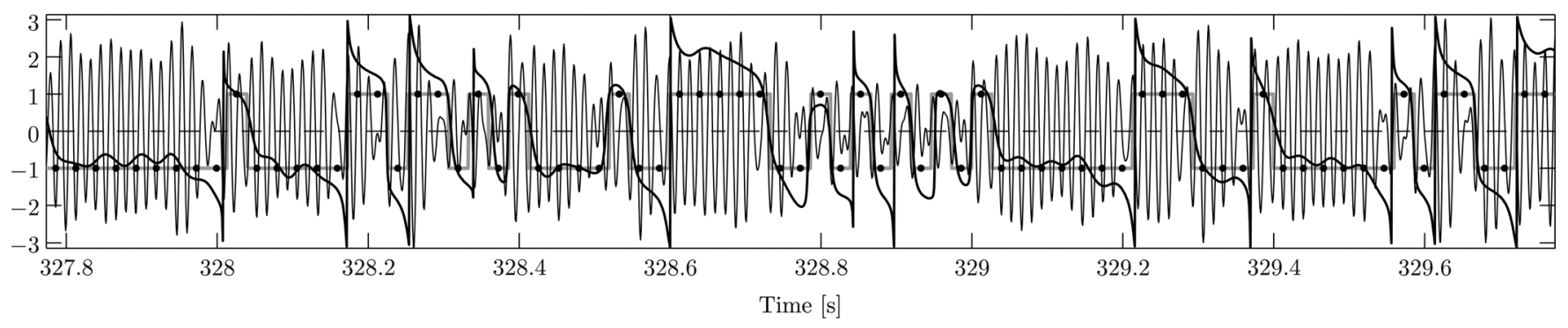

FIG. 2. Examples of T50 (top panel), T250 (middle panel), and T500 (bottom panel) modal pulse processing for $m=1$. The thin black line shows mode 1 amplitude $a_{1}(t)$; the thick black line shows the phase function $Y_{1}(t)$; the thick gray line is the idealized transmitted square wave (with unit amplitude); the black dots show the bits recovered from the phase function. The horizontal axis is absolute arrival time. The vertical axis on each panel shows phase function values $Y_{1}(t)$ (which vary between $-\pi$ and $\pi$ ), on which normalized modal amplitude arrivals $a_{1}(t)$ are superimposed in arbitrary units.

A different way to summarize our results is shown in Fig. 3. In that figure, in-phase components of discrete samples of $a_{m}(t)$ are plotted vs the corresponding quadrature components. In such constellation plots [see, e.g., Freitag and Stojanovic (2001) and Song et al. (2009)] good performance in recovery of a binary sequence corresponds to a clear separation between two sets of points; in our case the dividing line between + and - bits is the horizontal axis. If one focuses only on the position of points relative to that dividing line (ignoring the sometimes multimodal distributions), the exact same trends that were noted in our discussion of Table I are seen in Fig. 3.

The multimodal distributions in Fig. 3 require some explanation. The phase modulation angle of the transmitted $m$-sequence was $\vartheta_{0}=\tan ^{-1}(\sqrt{1023}) \approx 88.2^{\circ}$. Recall that each transmitted digit consists of two cycles of the $75 \mathrm{~Hz}$ carrier. Ideally, all transmitted digits have the same amplitude and the phase shifts instantaneously between $\pm \vartheta_{0}$. Thus, ideally any sequence of + and - bits corresponds to two-point constellation plots, with the two points falling almost on the vertical axis, one above and one below (and equidistant from) the horizontal axis. In reality, however, the phase of the source does not change instantaneously when transitioning between + and - bits, and full amplitude is realized only after multiple repetitions of a bit, e.g., ++
++ . Thus, for any physically realizable source (including of course the LOAPEX source) + bits in the sequence -+-+-+- will be noticeably different than the second, third, and fourth + bits in the sequence -++++ . We have confirmed this behavior using T50 mode 1 receptions. The exact response of the LOAPEX source is not known, but the qualitative features of the complicated distributions seen in Fig. 3, especially at the shorter ranges where SNR is highest, are not surprising. An example of imperfect detection is demonstrated in Fig. 4. Modal pulse processing for $m=1$ of another T50 reception is shown in the bottom panel. The upper panel is the same as the upper panel in Fig. 2 with expanded time axis. Three single-bit errors occurred around $31.50,31.87$, and $32.08 \mathrm{~s}$ within the reception interval shown. The erroneously detected bits are labeled with bold " $\times$ " symbols. Black lines and dots have the same meaning as in Fig. 2. In all three instances erroneously detected bits are isolated, i.e., surrounded by bits of the opposite sign.

\section{SNR AND ISI}

It is useful to identify the dominant factors that contribute to errors in extracted bit streams. In this section we consider the influence of SNR and modal pulse broadening (which leads to ISI) on BERs. SNR was estimated for every 
TABLE I. Summary of BERs observed in the LOAPEX transmissions from stations T50, T250, and T500 for each of the first 10 modes. The entries summarize the results obtained for individual transmissions, transmissions coherently averaged over 5 min, and transmissions coherently averaged over 15 min. The number of transmissions with BER $=0$, BER between $0 \%$ and $1 \%$, BER between $1 \%$ and $10 \%$, BER greater than $10 \%$, and the total number of transmissions are listed for each transmission scenario.

\begin{tabular}{|c|c|c|c|c|c|c|c|c|c|c|}
\hline \multirow[b]{3}{*}{ Mode } & \multirow{2}{*}{$\begin{array}{l}\text { Transmission station } \\
\text { Averaging times (min) }\end{array}$} & \multicolumn{3}{|c|}{ T50 } & \multicolumn{3}{|c|}{$\mathrm{T} 250$} & \multicolumn{3}{|c|}{ T500 } \\
\hline & & 0 & 5 & 15 & 0 & 5 & 15 & 0 & 5 & 15 \\
\hline & BERS & & & & & & & & & \\
\hline \multirow[t]{5}{*}{1} & 0 & 291 & 30 & 10 & 249 & 26 & 10 & 30 & 10 & 3 \\
\hline & $0 \%$ to $1 \%$ & 32 & 0 & 0 & 51 & 5 & 0 & 71 & 3 & 1 \\
\hline & $1 \%$ to $10 \%$ & 7 & 0 & 0 & 63 & 2 & 1 & 232 & 21 & 8 \\
\hline & $>10 \%$ & 0 & 0 & 0 & 0 & 0 & 0 & 129 & 8 & 2 \\
\hline & Total & 330 & 30 & 10 & 363 & 33 & 11 & 462 & 42 & 14 \\
\hline \multirow[t]{5}{*}{2} & 0 & 313 & 30 & 10 & 11 & 14 & 7 & 0 & 1 & 1 \\
\hline & $0 \%$ to $1 \%$ & 17 & 0 & 0 & 74 & 12 & 3 & 24 & 5 & 1 \\
\hline & $1 \%$ to $10 \%$ & 0 & 0 & 0 & 275 & 7 & 1 & 238 & 28 & 10 \\
\hline & $>10 \%$ & 0 & 0 & 0 & 3 & 0 & 0 & 200 & 8 & 2 \\
\hline & Total & 330 & 30 & 10 & 363 & 33 & 11 & 462 & 42 & 14 \\
\hline \multirow[t]{5}{*}{3} & 0 & 244 & 30 & 10 & 43 & 8 & 2 & 1 & 6 & 3 \\
\hline & $0 \%$ to $1 \%$ & 80 & 0 & 0 & 54 & 4 & 2 & 76 & 13 & 3 \\
\hline & $1 \%$ to $10 \%$ & 6 & 0 & 0 & 260 & 21 & 7 & 286 & 16 & 6 \\
\hline & $>10 \%$ & 0 & 0 & 0 & 6 & 0 & 0 & 99 & 7 & 2 \\
\hline & Total & 330 & 30 & 10 & 363 & 33 & 11 & 462 & 42 & 14 \\
\hline \multirow[t]{5}{*}{4} & 0 & 12 & 9 & 4 & 0 & 0 & 0 & 0 & 0 & 0 \\
\hline & $0 \%$ to $1 \%$ & 100 & 17 & 4 & 1 & 1 & 1 & 0 & 0 & 0 \\
\hline & $1 \%$ to $10 \%$ & 218 & 4 & 2 & 280 & 30 & 10 & 33 & 8 & 4 \\
\hline & $>10 \%$ & 0 & 0 & 0 & 82 & 2 & 0 & 429 & 34 & 10 \\
\hline & Total & 330 & 30 & 10 & 363 & 33 & 11 & 462 & 42 & 14 \\
\hline \multirow[t]{5}{*}{5} & 0 & 185 & 30 & 10 & 3 & 6 & 4 & 0 & 0 & 0 \\
\hline & $0 \%$ to $1 \%$ & 135 & 0 & 0 & 30 & 13 & 3 & 0 & 0 & 0 \\
\hline & $1 \%$ to $10 \%$ & 10 & 0 & 0 & 237 & 6 & 2 & 0 & 0 & 0 \\
\hline & $>10 \%$ & 0 & 0 & 0 & 93 & 8 & 2 & 462 & 42 & 14 \\
\hline & Total & 330 & 30 & 10 & 363 & 33 & 11 & 462 & 42 & 14 \\
\hline \multirow[t]{5}{*}{6} & 0 & 8 & 5 & 3 & 0 & 0 & 0 & 0 & 0 & 0 \\
\hline & $0 \%$ to $1 \%$ & 96 & 18 & 6 & 0 & 1 & 0 & 0 & 0 & 0 \\
\hline & $1 \%$ to $10 \%$ & 219 & 7 & 1 & 77 & 11 & 4 & 0 & 0 & 0 \\
\hline & $>10 \%$ & 7 & 0 & 0 & 286 & 21 & 7 & 462 & 42 & 14 \\
\hline & Total & 330 & 30 & 10 & 363 & 33 & 11 & 462 & 42 & 14 \\
\hline \multirow[t]{5}{*}{7} & 0 & 0 & 0 & 0 & 0 & 0 & 0 & 0 & 0 & 0 \\
\hline & $0 \%$ to $1 \%$ & 0 & 0 & 0 & 0 & 0 & 0 & 0 & 0 & 0 \\
\hline & $1 \%$ to $10 \%$ & 304 & 30 & 10 & 0 & 0 & 0 & 0 & 0 & 0 \\
\hline & $>10 \%$ & 26 & 0 & 0 & 363 & 33 & 11 & 462 & 42 & 14 \\
\hline & Total & 330 & 30 & 10 & 363 & 33 & 11 & 462 & 42 & 14 \\
\hline \multirow[t]{5}{*}{8} & 0 & 0 & 0 & 0 & 0 & 0 & 0 & 0 & 0 & 0 \\
\hline & $0 \%$ to $1 \%$ & 0 & 2 & 1 & 0 & 0 & 0 & 0 & 0 & 0 \\
\hline & $1 \%$ to $10 \%$ & 330 & 28 & 9 & 0 & 1 & 0 & 0 & 0 & 0 \\
\hline & $>10 \%$ & 0 & 0 & 0 & 363 & 32 & 11 & 462 & 42 & 14 \\
\hline & Total & 330 & 30 & 10 & 363 & 33 & 11 & 462 & 42 & 14 \\
\hline \multirow[t]{5}{*}{9} & 0 & 0 & 0 & 0 & 0 & 0 & 0 & 0 & 0 & 0 \\
\hline & $0 \%$ to $1 \%$ & 13 & 14 & 8 & 0 & 0 & 0 & 0 & 0 & 0 \\
\hline & $1 \%$ to $10 \%$ & 317 & 16 & 2 & 0 & 0 & 0 & 0 & 0 & 0 \\
\hline & $>10 \%$ & 0 & 0 & 0 & 363 & 33 & 11 & 462 & 42 & 14 \\
\hline & Total & 330 & 30 & 10 & 363 & 33 & 11 & 462 & 42 & 14 \\
\hline \multirow[t]{5}{*}{10} & 0 & 0 & 3 & 2 & 0 & 0 & 0 & 0 & 0 & 0 \\
\hline & $0 \%$ to $1 \%$ & 24 & 11 & 4 & 0 & 0 & 0 & 0 & 0 & 0 \\
\hline & $1 \%$ to $10 \%$ & 259 & 15 & 4 & 0 & 0 & 0 & 0 & 0 & 0 \\
\hline & $>10 \%$ & 47 & 1 & 0 & 363 & 33 & 11 & 462 & 42 & 14 \\
\hline & Total & 330 & 30 & 10 & 363 & 33 & 11 & 462 & 42 & 14 \\
\hline
\end{tabular}

transmission and every mode number from the ratio of modal amplitudes computed using active source data to modal amplitudes computed using recordings with the source turned off. The average SNR was estimated for each 5 min block of data. Quantification of pulse broadening and ISI is less obvious. To motivate the ISI measure that we 

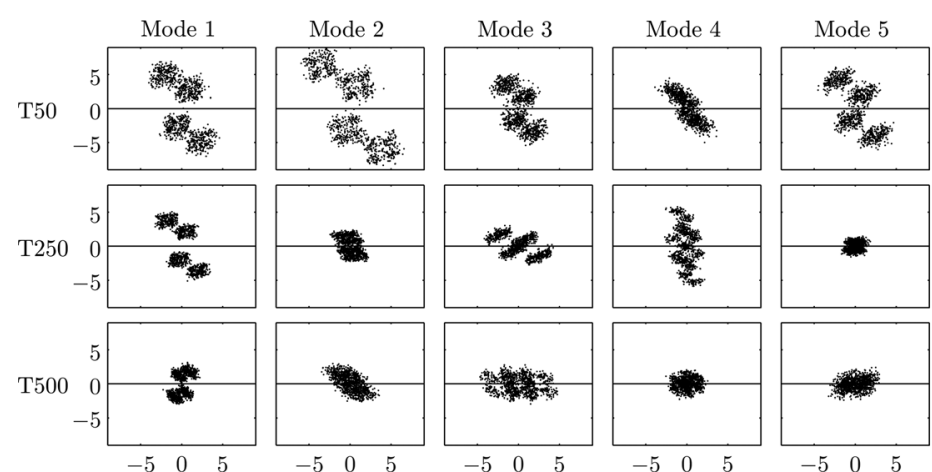
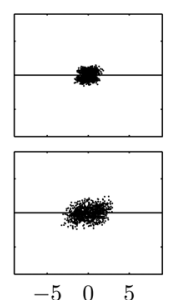
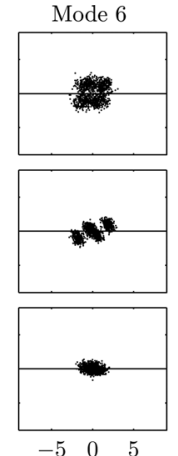
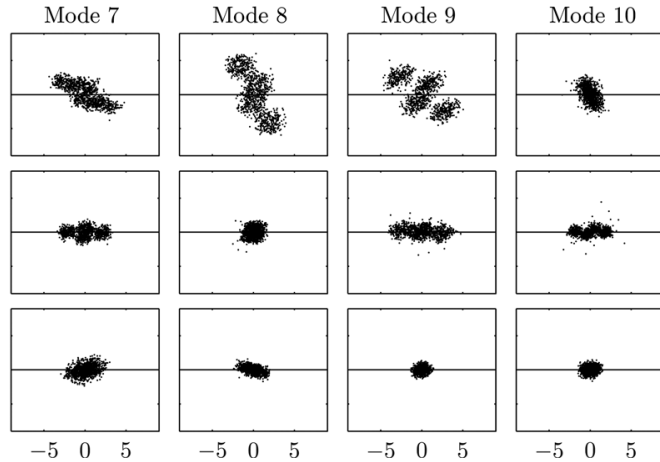
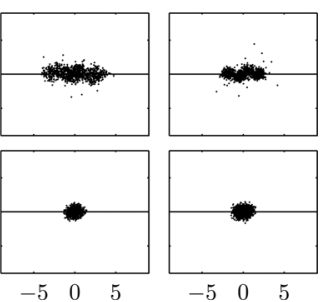

FIG. 3. Examples of T50 (top row), T250 (middle row), and T500 (bottom row) constellation plots for modes 1 through 10 . + and - bits are well-resolved if the horizontal axis separates the constellations into two well-defined groups, as is the case for modes 1, 2, and 3 at T50, and mode 1 at T250 and T500.

employ, we note first that there are three contributions to modal pulse time spreads (Udovydchenkov and Brown, 2008): The reciprocal bandwidth; a dispersive spreading term, and a scattering-induced spreading term. The three terms combine approximately in quadrature. The reciprocal bandwidth is the duration of a single digit of the transmitted sequence, which is unrelated to pulse broadening and ISI. In contrast, the dispersive and scattering terms lead to pulse broadening and ISI. (Recall that those terms were discussed earlier and that weakly dispersive modal pulses are special modal pulses for which both the dispersion and scattering terms are near zero.) Because the three contributions combine approximately in quadrature, it is natural to define, and use as a measure of anticipated ISI, the propagation-induced pulse broadening as $\Delta t=\sqrt{\Delta t_{\text {total }}^{2}-\Delta t_{\mathrm{bw}}^{2}}$. Here $\Delta t_{\text {total }}$ is the data-based estimate of modal group time spread [see Sec. IV (B) in Udovydchenkov et al. (2012) for details on estimation of $\Delta t_{\text {total }}$, and $\Delta t_{\mathrm{bw}}$ is the reciprocal bandwidth contribution to the total modal group time spread; the assumed numerical value of $\Delta t_{\mathrm{bw}}$ was $0.053 \mathrm{~s}$ (Udovydchenkov and Brown, 2008; Udovydchenkov et al., 2012). For communications applications a useful quantitative definition of a weakly dispersive modal pulse is one for which $\Delta t$ $\lesssim \Delta t_{\mathrm{bw}}$. Note that consistently with our definition of $\Delta t_{\mathrm{bw}}$, for the signal used in the experiment, $\Delta t_{\text {bw }}$ is approximately twice the duration of the transmitted digit $(0.0267 \mathrm{~s})$.

Figure 5 shows BER, SNR, and propagation-induced modal pulse spread for the first three modes as a function of experimental time. Each data point corresponds to a $5 \mathrm{~min}$ average. BER error bars correspond to one standard deviation. No error bars are shown for SNR and $\Delta t$. The plotted times are relative to the time of the first analyzed data file for each transmission type. Figure 5 shows that BERs tend to cluster in time, but the dependence of BER on SNR and $\Delta t$ is not clear in that figure. Figure 6 shows plots of BER vs SNR and BER vs $\Delta t$ for all individual receptions of the first three modes. That figure shows that BER generally decreases with increasing SNR, and that BER generally decreases with decreasing $\Delta t$. But there is a great deal of scatter in both plots so neither SNR nor $\Delta t$ alone can account for trends in BER. Instead, a combination of these factors-and possibly others-appear to be relevant. Consider now some special

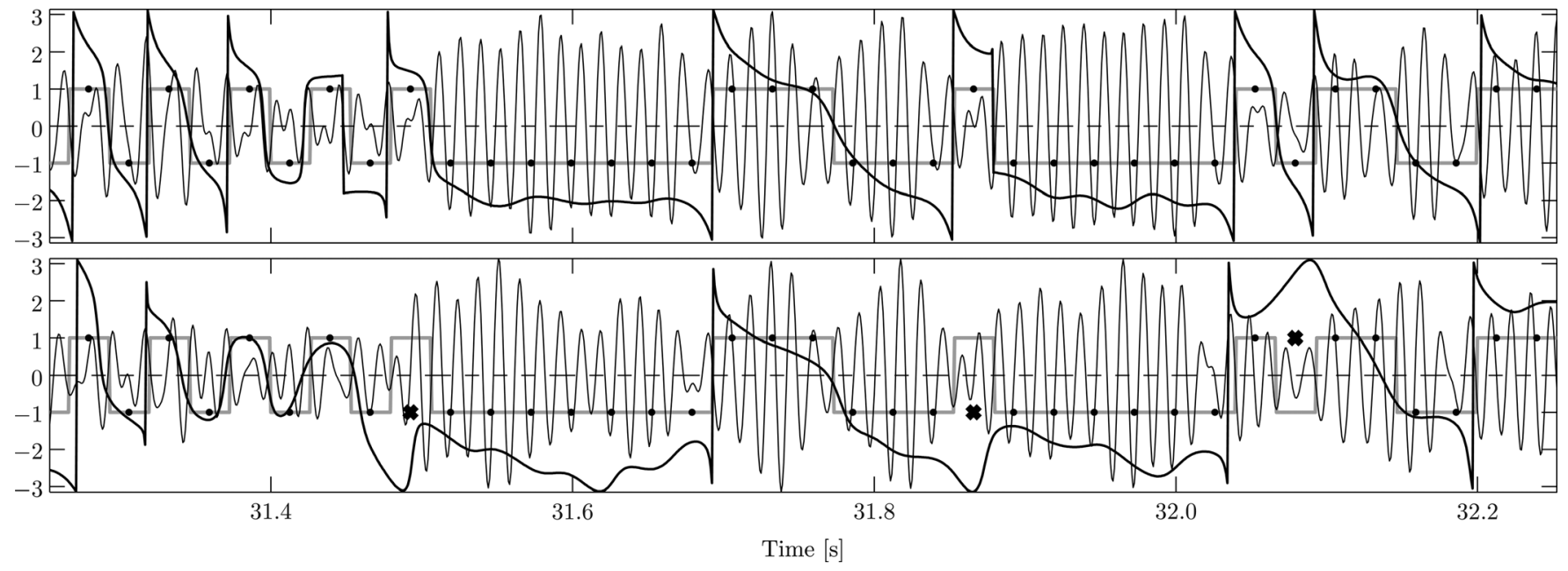

FIG. 4. Modal pulse processing for $m=1$ without errors (top panel) and with errors (bottom panel). The top panel is the same as the upper panel of Fig. 2 with expanded time axis and is shown here for convenience. The bottom panel curves show processing steps for another T50 transmission recorded during hour 8 (see Fig. 5 for BER estimates at this hour). Three single-bit errors occurred during the reception window shown. The erroneously identified bits are plotted with bold x symbols. 
T50

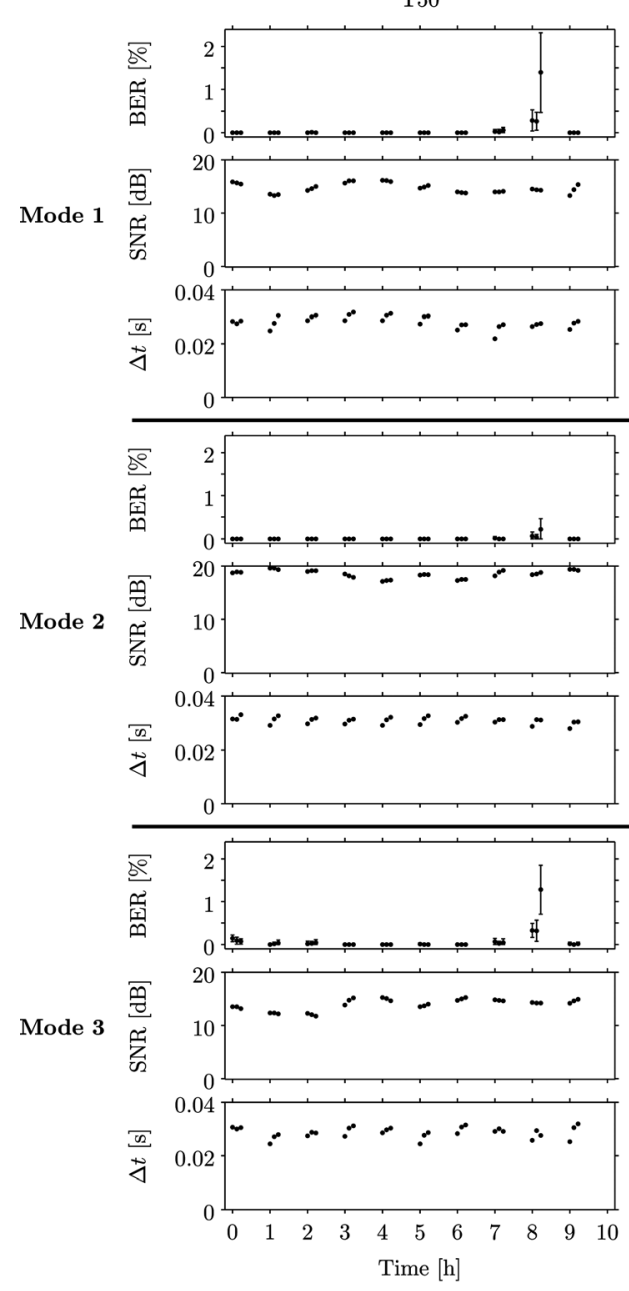

$\mathrm{T} 250$
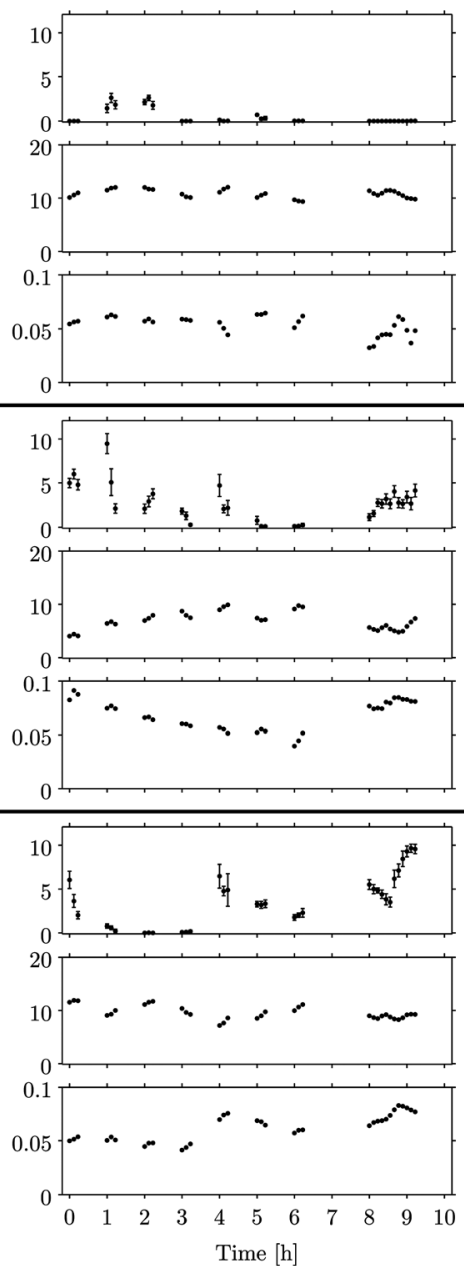

T500
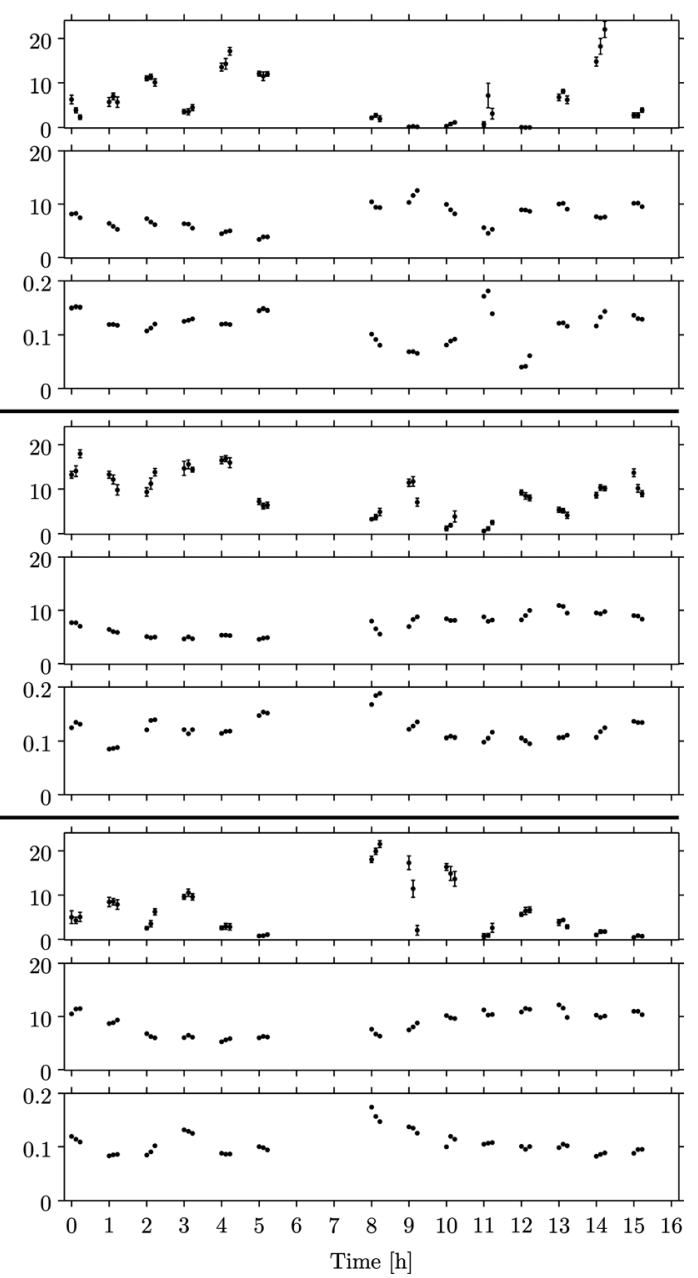

FIG. 5. Data-based estimates of BER, SNR, and modal pulse spread $(\Delta t)$ as a function of experimental time for mode numbers 1 , 2, and 3. Mean values and one standard deviation are shown for BER estimates. Only mean values are shown for SNR and $\Delta t$ estimates.

cases shown in Fig. 5. At T50 BERs are near zero everywhere except during transmission hour 8 , where a few transmissions have BERs between $1 \%$ and $10 \%$. At T250 modes 2 and 3 have high BERs during hours 8 and 9; that is likely due to the absence of mooring navigation data during those hours. At T50 BERs are lower during hour 8 for mode 2 than for mode 1 because mode 2 is more strongly excited than mode 1. At T250 the SNR for mode 2 is lowest for the earliest and latest transmissions; correspondingly, BERs are highest during the first few and last few hours. Similar trends can be noted between $\Delta t$ and BER: Generally, an increase in $\Delta t$ leads to a higher BER. An example is mode 3 at T500, where both $\Delta t$ and BER reach maxima at hour 8 . Another example is mode 3 at T250 for hours 4 through 6; with SNR nearly constant, BER decreases with decreasing $\Delta t$.
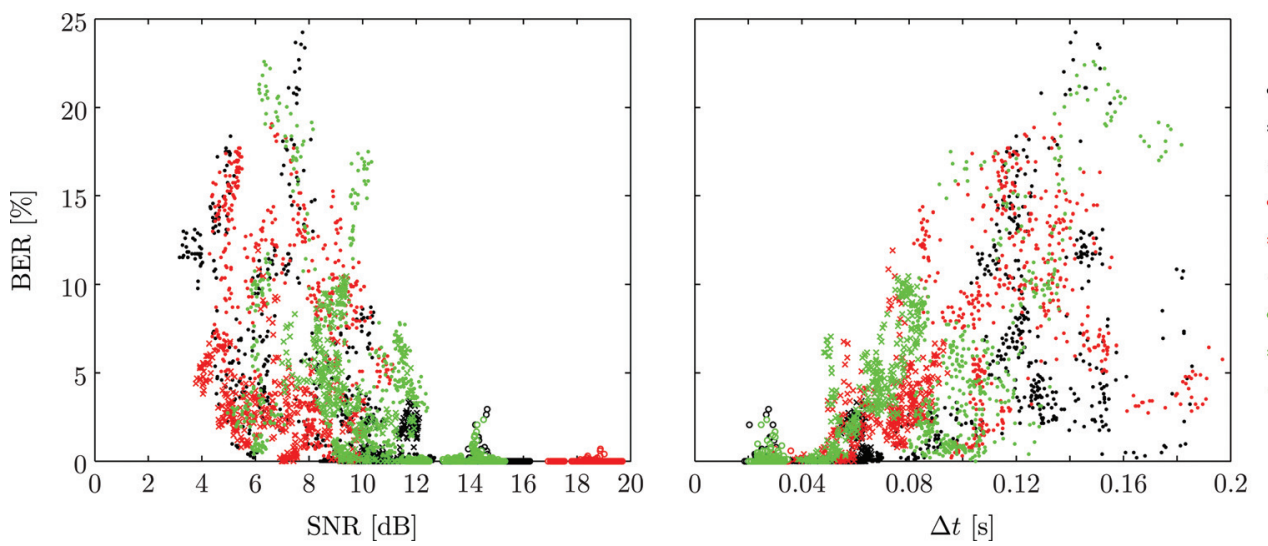

T50 Mode 1

T250 Mode 1

T500 Mode 1

T50 Mode 2

T250 Mode 2

T500 Mode 2

T50 Mode 3

T250 Mode 3

T500 Mode 3

FIG. 6. BER vs SNR and BER vs $\Delta t$ (propagation-induced modal pulse spread, as described in the text) for mode numbers 1, 2, and 3 for all unaveraged T50, T250, and T500 receptions. 


\section{CONCLUSIONS}

Motivated by potential communications applications, the propagation of weakly dispersive modal pulses in a deep ocean sound channel has been investigated experimentally. It has been shown that, without adaptive equalization, channel impulse response estimation, or any form of receiver training, communication using weakly dispersive modal pulses at a rate of $37.5 \mathrm{bps}$ is stable at a range of $50 \mathrm{~km}$, generally works at $250 \mathrm{~km}$, and sometimes works at $500 \mathrm{~km}$. For mode 1 at $500 \mathrm{~km}$ range, $6.5 \%$ of transmissions had no errors, and $72 \%$ of transmissions had BERs less than $10 \%$. At 250 and $500 \mathrm{~km}$ range, coherent averaging over 5 or $15 \mathrm{~min}$ led to a reduction of BERs. Low BERs were shown to be associated with high SNR and small propagation-induced modal pulse time spread (which leads to low ISI). The results presented are in good qualitative agreement with the theoretically predicted dependencies on mode number and range that we have noted.

From a communications perspective, the results reported here serve as a "proof of concept" rather than an attempt to develop an optimal processing scheme. In communications applications the results presented here could almost certainly be improved significantly by incorporating some form of channel equalization in the signal processing performed. Consistent with our more narrow focus of elucidating the physics of weakly dispersive modal pulses in a deep ocean sound channel, we have chosen not to consider equalization in this paper. If equalization is performed after extracting modal pulses, it is natural to focus on those mode numbers corresponding to weakly dispersive modal pulses; the demands on the equalization filter are lowest for that special class of modal pulses.

\section{ACKNOWLEDGMENTS}

This work was supported by the Office of Naval Research, Code 322, Grant Nos. N00014-06-1-0245, N00014-08-1-0195, and N00014-11-1-0194.

Brown, M. G., and Udovydchenkov, I. A. (2013). "Underwater communication using weakly dispersive modal pulses," Acoust. Phys. 59, 533-538.

Freitag, L., and Stojanovic, M. (2001). "Basin-scale acoustic communication: A feasibility study using tomography m-sequences," OCEANS, MTS/ IEEE Conference and Exhibition, Vol. 4, pp. 2256-2261.

Makarov, D., Prants, S., Virovlyansky, A. L., and Zaslavsky, G. (2010). Ray and Wave Chaos in Ocean Acoustics (World Scientific, Hackensack, NJ), 389 p.

Mercer, J. A., Andrew, R. K., Howe, B. M., and Colosi, J. A. (2005). "Cruise report: Long-range ocean acoustic propagation experiment (LOAPEX)," Technical Report APLUW TR0501, Applied Physics Laboratory, University of Washington, Seattle, WA.

Mercer, J. A., Colosi, J. A., Howe, B. M., Dzieciuch, M. A., Stephen, R., and Worcester, P. F. (2009). "LOAPEX: The long-range ocean acoustic propagation experiment," IEEE J. Ocean. Eng. 34, 1-11.

Morozov, A. K., Preisig, J. C., and Papp, J. (2008). "Modal processing for acoustic communications in shallow water experiment," J. Acoust. Soc. Am. 124, EL177-EL181.

Oppenheim, A., Schafer, R. W., and Buck, J. R. (1999). Discrete-Time Signal Processing (Prentice-Hall, Inc., Upper Saddle River, NJ), Chap. 5.6, pp. 280-291.

Parks, T. W., and Burrus, C. S. (1987). Digital Filter Design (John Wiley and Sons, Inc., New York), Chap. 7, pp. 161-171 and 213-218.

Song, H. C., and Dzieciuch, M. (2009). "Feasibility of global-scale synthetic aperture communications," J. Acoust. Soc. Am. 125, 8-10.

Song, H. C., Kuperman, W. A., and Hodgkiss, W. S. (2009). "Basin-scale time reversal communications," J. Acoust. Soc. Am. 125, 212-217.

Udovydchenkov, I. A., and Brown, M. G. (2008). "Modal group time spreads in weakly range-dependent deep ocean environments," J. Acoust. Soc. Am. 123, 41-50.

Udovydchenkov, I. A., Brown, M. G., Duda, T. F., Mercer, J. A., Andrew, R. K., Worcester, P. F., Dzieciuch, M. A., Howe, B. M., and Colosi, J. A. (2012). "Modal analysis of the range evolution of broadband wavefields in the North Pacific Ocean: Low mode numbers," J. Acoust. Soc. Am. 131, 4409-4427.

Virovlyansky, A. L., Kazarova, A. Yu., and Lyubavin, L. Ya. (2007). "Statistical description of chaotic rays in a deep water acoustic waveguide," J. Acoust. Soc. Am. 121, 2542-2552. 